\title{
Wochenendgeburten und Kaiserschnittraten in der Schweiz
}

Michael Singer,

Präsident der Gynäkologischen Vereinigung des Kantons Zürich
Prof. Lerchl aus Bremen kommentiert pointiert sein Zahlenmaterial [1], gemäss dem in der Schweiz prozentual etwas weniger Kinder an Wochenenden geboren werden, wobei dieser Effekt an kleineren Geburtskliniken ausgeprägter zu sein scheint. Er leitet daraus die wahrlich abenteuerliche Anschuldigung ab, dass kleinere Geburtskliniken die Schwangeren aus finanziellen Gründen zum Kaiserschnitt drängen könnten. Der Kurzbeitrag ist gespickt mit unbelegten plakativen Behauptungen wie «Kaiserschnitte sind nicht nur teurer als vaginale Geburten, sondern sind auch mit einem höheren Risiko für Mutter und Kind verbunden».

Es darf gerätselt werden, weshalb der Zoologieprofessor einer privaten deutschen Universität (persönlicher Forschungsschwerpunkt: die biologische Uhr) derart schwerwiegende Mutmassungen über die Geburtshilfe in der Schweiz anstellt und in unserer Schweizerischen Ärztezeitung abdrucken lässt. Die Datenbasis der Lerchlschen Behauptungen ist so schmal und der methodische Ansatz so fragwürdig, dass sich aus der Optik des praktizierenden Geburtshelfers die folgenden Feststellungen aufdrängen.

\section{Hat die Verteilung der Geburten über die Wochentage eine medizinische oder gesundheitspolitische Bedeutung?}

Es ist plausibel, dass die relative Untervertretung der Wochenendgeburten mit der Zunahme primärer (geplanter) und sekundärer (notfallmässiger) Kaiserschnitte zusammenhängt. Sowohl bei einem geplanten Kaiserschnitt als auch bei einer medizinisch begründeten Geburtseinleitung (aus der sich ein Notfallkaiserschnitt ergeben kann) besteht ein zeitlicher Spielraum, weshalb Geburtskliniken die Plansectios und Geburtseinleitungen schwergewichtig unter der Woche ansetzen. Dies ist weder verwerflich noch neu, sondern zeugt von einem verantwortungsbewussten Umgang mit den beschränkten personellen Ressourcen und entlastet die Wochenendequipen von vorhersehbaren Engpässen, was sich positiv auf die Patientensicherheit auswirkt. Wie viele Kollegen mit ausgelasteten Praxen würde ich gern schwierige Geburtseinleitungen bewusst auf das freie Wochenende legen (weil dann der Sprechstundenbetrieb weniger durch- einanderkommt), nehme dabei aber selbstverständlich auf die Wochenendkapazitäten der Kliniken Rücksicht.

Falls der Lerchlsche Effekt an kleinen Geburtskliniken stärker auftritt, so darf daraus keine eindeutige Interpretation und schon gar nicht die böswillige Unterstellung eines finanziell motivierten Verhaltens abgeleitet werden. Die notwendigen statistischen Zusatzinformationen betreffend Patientenmix, organisatorische und infrastrukturelle Gegebenheiten (Einfluss von Intensivstationen und tertiärer Zentrumsfunktion bei grösseren Kliniken) sind schlicht nicht vorhanden. Anhand statistischer Assoziationen politisch gefärbte Kausalzusammenhänge zu suggerieren ist ungefähr auf demselben Niveau wie die berühmte Schlussfolgerung, dass zwischen der Zunahme der Störche und den steigenden Geburtenzahlen ein Kausalzusammenhang besteht. Lerchl beleidigt zudem die autonome und wohlinformierte schwangere Frau, wenn er glaubt, dass sie sich mit fadenscheinigen Argumenten zu einem Kaiserschnitt überreden liesse, um die Spitalfinanzen aufzubessern.

Zusammenfassend haben die Lerchlschen Feststellungen - und seien sie noch so dramatisch eingefärbt - weder einen Neuigkeitswert, noch leitet sich daraus ein Handlungsbedarf ab.

\section{Sind Kaiserschnitte gefährlicher und teurer?}

Lerchl bedient sich des auch hierzulande von gewissen Krankenkassen und Politikern bemühten Klischees, dass zu viele gefährliche und teure Kaiserschnitte stattfinden. Tatsache ist, dass die geplante (primäre) Sectio heute bezüglich Komplikationen für Mutter und Kind genauso gut dasteht wie die vaginale Geburt [2-4], wobei noch nicht berücksichtigt ist, dass die vaginale Geburt in mindestens 10\% vaginal-operativ (Vakuum, Forceps) und weiteren 10\% als sekundäre Notfallsectio endet. Unbestritten ist auch, dass die sekundäre (notfallmässige) Sectio für Mutter und Kind am belastendsten, für das Geburtserlebnis am schlechtesten und für die Geburtsklinik am kostspieligsten ist. Daraus leitet Prof. Husslein aus Wien die logische Forderung $a b$, dass heute keineswegs die tiefe Kaiserschnittrate an sich, sondern die tiefe Rate 
Literatur

1 Lerchl A. Wochenendgeburten und Kaiserschnittraten in der Schweiz. Schweiz Ärztezeitung. 2007;88(40):1676-7.

2 Haller U, Hepp H., Winter R. Sectio nach Wunsch oder elektive Sectio: Aufforderung zum Umdenken. Gynäkologisch-geburtshilfliche Rundschau. 2002;42:1-3.

3 Husslein P, Langer M. Elektive Sectio versus vaginale Geburt ein Paradigmenwechsel in der Geburtshilfe? Gynäkologe. 2000; 33:849-56.

4 Hänggi-Bally D, Heinzl S. Ist die natürliche Geburt «out»? Schweiz Med Forum. 2004;4(49):1244-51.

5 Bundesamt für Statistik. Gebären in Schweizer Spitälern. BFS:

Neuenburg; 2007. www.bfs. admin.ch/bfs/portal/de/index/ news/publikationen. Document. 90668.pdf. sekundärer Sectiones als Qualitätsmerkmal einer Geburtsklinik zu gelten hat [3]

Bei der Behauptung, ein Kaiserschnitt sei fast doppelt so teuer, bezieht sich Lerchl auf die lesenswerte BFS-Statistik [5]. Dabei ignoriert er völlig, dass dort nicht reale Frankenbeträge, sondern virtuelle Kostengewichte gemäss AP-DRG-Fallgruppen verglichen werden, wobei - und dies ist ein methodisches Grundproblem aller heute verfügbaren Daten - die primären und die sekundären Sectiones in einen Topf geworfen werden, obwohl es hinsichtlich Nutzung der Infrastruktur und Hospitalisationsdauer grundverschiedene Situationen sind.

Zur Bedeutung der Patientenautonomie und des gesellschaftlich-juristischen Drucks auf die Geburtshelfer, eine Null-Risiko-Geburtsmedizin zu betreiben, zitiere ich die Zusammenfassung von Haller [2]: «Die zunehmende Gewichtung der Autonomie und das Recht auf Selbstbestimmung der Frau führen [...] zu einer grundlegenden Veränderung der Arzt-Patienten-Beziehung. Langzeitfolgen nach vaginaler Geburt, wie Verletzung des Beckenbodens mit funktioneller Beeinträchtigung sowie Störungen der Sexualfunktion, aber auch Überlegungen zur Sicherheit des Kindes gewinnen für die Schwangere zunehmende Bedeutung und müssen von ärztlicher
Seite ernst genommen werden. Obwohl genaue Berechnungsgrundlagen nicht vorliegen, wird ein globaler Kostenvergleich zwischen einer vaginalen Geburt und einer elektiven Sectio mit Berücksichtigung der Langzeitfolgen kaum eigentliche Unterschiede aufweisen, weshalb es für die Versicherer keine Berechtigung gibt, die Kostenübernahme zu verweigern.»

Zusammenfassend ist die in allen Industrieländern zu beobachtende Zunahme der Kaiserschnittrate ein vielschichtiges geburtsmedizinisches, gesellschaftliches und haftungsrechtliches Phänomen. Lerchl ignoriert den fundamentalen medizinischen und wirtschaftlichen Unterschied zwischen primären und sekundären Kaiserschnitten. Seine künstliche Aufregung über «Vermeidung von Wochenendgeburten» wird der Komplexität des Themas in keiner Weise gerecht.

Zum Schluss kann ich Prof. Lerchl beruhigen: In meiner persönlichen Statistik der vaginalen Geburten der letzten zehn Jahre fanden je 15,5\% an einem Samstag oder Sonntag statt, d.h., die Wochenendgeburten waren leicht übervertreten. Diese biologische Uhr scheint also noch zu funktionieren.

\section{Replik}

\section{Alexander Lerchl}

Korrespondenz:

Prof. Dr. Alexander Lerchl Jacobs University Bremen School of Engineering and Science, Research II

Campus Ring 6

D-28759 Bremen

Tel. +494212003241

Fax +49421200493241

a.lerchl@jacobs-university.de
Als Professor für Biologie (die ja den Menschen mit einschliesst) und während meiner vorherigen, über 10jährigen Tätigkeit im Institut für Reproduktionsmedizin der Universität Münster beschäftige ich mich seit vielen Jahren mit Einflüssen auf die menschliche Fortpflanzung. Der von mir 2005 aufgezeigte Trend zu immer weniger Wochenendgeburten in Deutschland [1] war für mich Anlass, auch in anderen Ländern nach solchen Veränderungen zu suchen und zu ergründen, welche Faktoren hierfür verantwortlich sind. Die von Frau Reinhard und mir analysierten Daten aus der Schweiz [2] umfassen die Anzahl und Wochentagsverteilung von insgesamt knapp 3 Millionen Geburten aus 37 Jahren, sind also kaum als «schmale Datenbasis» zu bezeichnen, sondern stellen die bislang grösste Studie zu diesem Thema dar. Es war überdies keineswegs unsere Absicht, «politisch gefärbte
Kausalzusammenhänge zu suggerieren» oder «böswillige Unterstellungen» abzuleiten, wie Herr Dr. Singer meint, sondern lediglich auf der Datenbasis beruhende Schlussfolgerungen zu ziehen.

Die Schweizer Daten wurden im übrigen insgesamt analysiert und keineswegs hinsichtlich der Grösse der Geburtskliniken, wie Herr Dr. Singer behauptet. Diese Fragestellung und mögliche Unterschiede zwischen den Schweizer Kantonen sowie der mögliche Einfluss des Versichertenstatus der Schwangeren wären allerdings sicherlich eine eigene Untersuchung wert.

Der «verantwortungsbewusste Umgang mit beschränkten personellen Ressourcen» am Wochenende und die daraus abgeleitete Folge, «Plansectios und Geburtseinleitungen schwergewichtig unter der Woche» anzusetzen, bestätigt die in unserer Veröffentlichung beschriebene 
Literatur

1 Lerchl A. Where are the Sunday babies? Observations on a marked decline in weekend births in Germany. Naturwissenschaften. 2005;50:592-4.

2 Lerchl A, Reinhard S. Where are the Sunday babies? II. Declining weekend birth rates in Switzerland. Naturwissenschaften. 2007. http://dx.doi.org/10.1007/ s00114-007-0305-4.

3 Lutz U, Kolip P. Die GEK-Kaiserschnittstudie. St. Augustin: Asgard; 2007. www.asfg.unibremen.de/doc/GEK-Kaiser schnittstudie_4.4.2006.pdf.
Vermutung [2] und unterstreicht trefflich die verdrehte Logik, einen wissentlich verursachten Notstand (geringe Wochenendkapazität) mit einer vermeintlich unausweichlichen Lösung (dann eben nicht am Wochenende) zu beantworten. Sie erklärt aber nicht, warum es in der Schweiz (wie auch in vielen anderen Ländern) immer weniger Geburten am Wochenende und immer mehr unter der Woche gibt (Abb. 1). Hier sind die von uns gefundenen statistischen Zusammenhänge von Sectios (sowohl Plan- als auch Notfallsectios) und Vermeidungsraten von Geburten am Wochenende hochinteressant, da sich aus ihnen stichhaltig ergibt, dass die steigende Kaiserschnittrate diesen Trend (mit)verursacht.

Noch interessanter und völlig unerwartet war der von uns gefundene, fast lineare Zusammenhang zwischen der Anzahl Geburten pro Jahr und der Vermeidung von Wochenendgeburten (Abb. 2): Je weniger Geburten in einem Jahr stattfanden, um so mehr Geburten fanden unter der Woche statt und um so weniger am Wochenende. Dieser Zusammenhang und die unbestritten wesentlich höheren Kosten für Sectios bei unbestritten niedrigerem Zeitaufwand legen die Vermutung nahe, dass diese Kosten keine unbedeutende Rolle spielen.

Dr. Singer führt aus, dass die «sekundäre (notfallmässige) Sectio für Mutter und Kind am belastendsten, für das Geburtserlebnis am schlechtesten und für die Geburtsklinik am kostspieligsten ist». In unserer Veröffentlichung [2] haben wir ebenfalls zeigen können, dass die Vermeidungsraten von Geburten am Wochenende nicht nur durch primäre, sondern auch durch sekundäre Sectios verursacht werden, da auch deren Anzahl stetig zunimmt. Häufig werden solche sekundären Sectios durch Einleitungen verursacht, die auch in der Schweiz zunehmen.

Eine im letzten Jahr veröffentlichte Studie aus Deutschland [3] hat anhand von Befragungen von über 1300 Frauen, die per Kaiserschnitt entbunden wurden, ergeben, dass lediglich 2\% von ihnen sich vor der Geburt für einen Kaiserschnitt entschieden haben. $60 \%$ erklärten hingegen, dass ihnen von ärztlicher Seite dazu geraten wurde. Wie das in der Schweiz aussieht, wäre ebenfalls eine eigene Untersuchung wert.

Die Schlussbemerkung von Dr. Singer widerspricht keineswegs unseren Befunden, da wir den Einfluss von Sectios untersucht haben, nicht die Verteilung von vaginalen Geburten.

\section{Abbildung 1}

Der Rückgang der Wochenendgeburten in der Schweiz von 1969 bis 2005. Zum Vergleich ist die Zunahme der Geburten unter der Woche (mittwochs) angegeben [2] http://dx.doi.org/10.1007/s00114-007-0305-4. (c) Springer

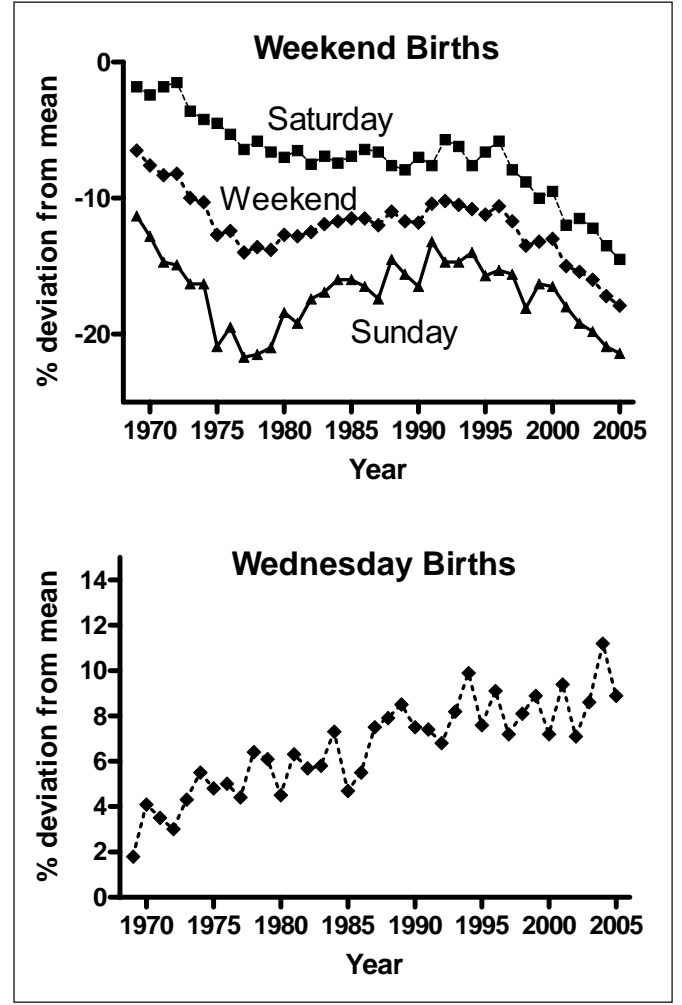

\section{Abbildung 2}

Vergleich der Anzahl Geburten (x-Achse) und Vermeidung von Wochenendgeburten ( $y$-Achse). Der Zusammenhang ist hochsignifikant $(p<0,001)[2]$. http://dx.doi.org/ 10.1007/s00114-007-0305-4. @ Springer

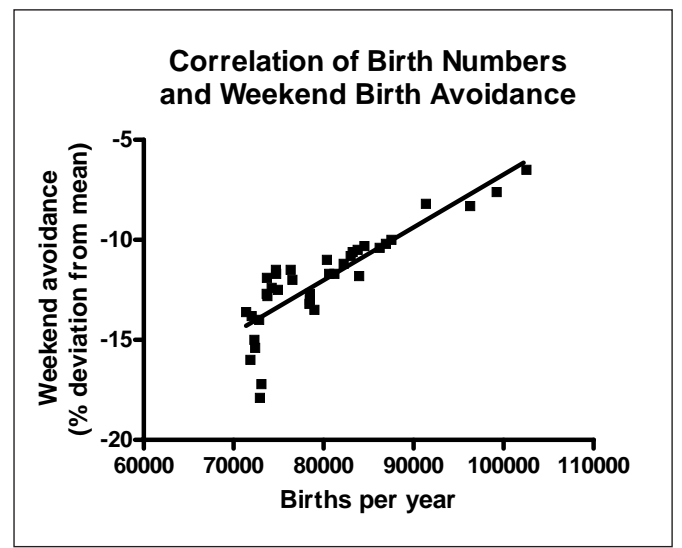

